\title{
Электронная ионизация молекул цитозина
}

\author{
"М. И. Шафраньош", М. Zapotokováb М. И. Суховия", \\ В. И. Петруляк ${ }^{\mathrm{a}}$, И. И. Шафраньош ${ }^{\mathrm{a}}$ \\ ${ }^{a}$ Ужгородский Национальный университет, \\ 2. Ужсгород, 88000, Украина, "e-mail: miroshafr@gmail.com \\ ${ }^{b}$ Прешовский университет, г. Прешов, 08001, Словакия \\ Поступила в редакцию 21.09.2020 \\ После доработки 30.11.2020 \\ Принята к публикации 30.11 .2020
}

\begin{abstract}
Методом пересекающихся электронного и молекулярного пучков и квантово-химическим методом AM1 выполнены исследования фрагментационных процессов в молекуле цитозина, инициированных электронным ударом. Приведены вероятные схемы фрагментации цитозина, абсолютные величины парциальных сечений ионизации и их энергетические зависимости. Указано, что величина суммарного сечения образования положительно заряженных фрагментов в 2,9 раза превышает сечения образования молекулярного иона. Проведен расчет параметров молекулы цитозина и ее ионов.
\end{abstract}

Ключевые слова: вторичные электроны, цитозин, электронный удар, сечения ионизации, энергетические зависимости, схемы фрагментации, молекулярный ион

УДК 539.19

https://doi.org/10.52577/eom.2021.57.2.42

\section{ВВЕДЕНИЕ}

Основания нуклеиновых кислот (НК) цитозин, аденин, тимин, урацил и гуанин относятся к числу важнейших биомолекул. Их исключительная биологическая значимость дала мощный толчок для системных исследований неупругих взаимодействий этих молекул с вторичными электронами, которые образуются в значительном количестве в веществе при воздействии излучения различных видов ( $\gamma$-лучи, электроны, ионы и т.д.) [1-6]. Особая роль в этих взаимодействиях отводится процессам ионизации, которые начинают цепь деструктивных изменений на молекулярном уровне биоструктур. По нашему мнению, такие исследования также представляют интерес для решения проблемы абиогенного происхождения жизни на Земле и определения возможных причин дисфункции механизмов узнавания на макромолекулярном уровне [7, 8]. В последнее время возникает вопрос, как адсорбция биомолекул в пористые поверхности твердого тела может защитить их от деградации в космической среде [9].

При энергиях ионизирующих электронов, превышающих энергию ионизации молекулы, вероятно образование иона в электронновозбужденном состоянии. Диссипация энергии этого состояния главным образом проходит двумя путями - радиационным, с изменением электронной конфигурации, либо нерадиационным за счет пересечения потенциальных поверхностей. Итак, возбужденный ион может перейти в основное состояние с избытком колебательной энергии. В то же время при пересечении потенциальных поверхностей переход иона в основное состояние не является единственно возможным. Если энергия возбужденного состояния иона является достаточной для его распада, то появляется возможность его фрагментации из этого состояния. В соответствии с классическими схемами фрагментации [10], построенными на основании массспектрометрических исследований сложных молекул, под действием электронного удара в молекуле происходит двухэлектронный переход. В результате этого образуются возбужденные положительные молекулярные ионы (т.н. материнские ионы), которые имеют большой избыток энергии. В дальнейшем материнские ионы распадаются, производя при этом заряженные и нейтральные фрагменты. Распад может происходить в нескольких направлениях и до тех пор, пока внутренняя энергия заряженного фрагмента будет достаточной для дальнейшей фрагментации. В работах [11-13] детально были рассмотрены фрагментационные процессы в молекулах азотистых оснований НК - тимина, урацила, аденина и гуанина под действием электронного удара. В данном исследовании представлены результаты о фрагментации молекул цитозина.

\section{ЭКСПЕРИМЕНТ И МЕТОД РАСЧЕТА}

Экспериментальные исследования были выполнены в условиях пересекающихся пучков 
электронов и молекул цитозина. Техника и методика эксперимента подробно описаны в нашей предыдущей работе [13]. Пучок исследуемых молекул формируется с помощью термического эффузионного источника многоканального типа и системы коллимирующих щелей. Температура эффузионного источника с препаратом цитозина в процессе измерения не превышала 405K, что является ниже температуры его деградации. Препарат для исследований изготовлен компанией SigmaAldrich (чистота 99\%).

В качестве источника электронов использовалась пятиэлектродная пушка с катодом, изготовленным из торированного вольфрама. Электроны пучка, прошедшие область столкновений, улавливались цилиндром Фарадея, который находился под положительным + потенциалом. Измерения проводились при силе тока пучка электронов $\sim 5 \times 10^{-6}$ А и энергетической неоднородности электронов на половине высоты их энергетического распределения $\Delta E_{1 / 2} \sim 0,3$ эВ. Электронная пушка размещалась в продольном магнитном поле с индукцией $B=1,2 \times 10^{-2}$ Тл. Калибровка энергетической шкалы электронов проводилась согласно резонансным пикам образования ионов $\mathrm{SF}^{-6}$, энергетическое положение которого определяло ноль шкалы.

Ионы, образовавшиеся в области пересечения электронного и молекулярного пучков, вытягивались электрическим полем и направлялись для масс-спектрометрического анализа $\mathrm{m} / \mathrm{z}$ ( $m$ и $z$ - соответственно масса и заряд иона). Для этой цели были использованы масс-спектрометр МИ 1201 (для записи масс-спектров) и лабораторный макет масс-спектрометра с отклонением ионов на $180^{\circ}$ (для измерений энергетических зависимостей парциальных сечений ионизации). Экспериментальные измерения осуществлялись в два этапа: на первом этапе записывались массспектры молекул при энергии бомбардирующих электронов 95 эВ и проводилась идентификация линий масс-спектров; на втором - изучались энергетические зависимости сечений образования заряженных фрагментов молекулы цитозина с выбранным отношением $m / z$. Абсолютные величины парциальных сечений ионизации определялись путем их нормирования на абсолютные величины полных сечений ионизации, определенные ранее в работе [14], следующим образом. Очевидно, что суммарный ток образованных ионов равен:

$$
i_{\Sigma}=i_{1}+i_{2}+\ldots+i_{n}
$$

где $i_{\Sigma}-$ суммарный ионный ток; $i_{n}-$ ток ионов определенного фрагмента.
В то же время:

$$
\begin{aligned}
& i_{\Sigma}=i_{e} n \sigma_{\Sigma} l, \\
& i_{n}=i_{e} n \sigma_{n} l
\end{aligned}
$$

где $i_{e}-$ ток бомбардирующих электронов; $n$ - концентрация молекул в области пересечения электронного и молекулярного пучков; $l$ - путь электронов в молекулярном пучке; $\sigma_{\Sigma} i \sigma_{n}-$ соответственно полное и парциальное сечения ионизации.

Таким образом, исходя из вышеприведенного следует:

$$
\sigma_{n}=\frac{\sigma_{\sum} i_{n}}{i_{\Sigma}}
$$

Учитывая, что ионный ток пропорционален площади масс-спектрометрического пика в массспектре, для искомого парциального сечения получим:

$$
\sigma_{n}=\frac{\sigma_{\sum} S_{n}}{\sum_{S_{i}}},
$$

где $S_{n}-$ площадь $n$-го пика в масс-спектре; $\sum S_{i}$ - суммарная площадь всех пиков в массспектре.

Приведенные в работе данные о сечении образования положительных ионов получены путем усреднения результатов пяти измерений. Относительные погрешности измерений составляют: $12 \%$ - для энергетических зависимостей сечений ионизации; $21 \%$ - для абсолютных величин.

Для теоретического моделирования процессов фрагментации молекулы цитозина под влиянием электронного удара был использован квантовохимический метод АM1 [15, 16], входящий в пакет программ НyperChem 8.1 [15]. Пакет содержит программы, обеспечивающие возможность квантово-химического расчета химических частиц вещества методами неэмпирической и полуэмпирической квантовой химии. Метод AM1 является улучшением метода MNDO. Он позволяет проводить более качественные расчеты электронной структуры, полной энергии теплоты образования частиц и оптимизировать их геометрию. В проведенных расчетах AM1 использовался в режиме оптимизации всех структурных параметров с нормой градиента $<0,01$.

\section{РЕЗУЛЬТАТЫ И ОБСУЖДЕНИЕ}

Масс-спектр цитозина при энергии бомбардирующих электронов 95 эВ приведен на рис. 1, 


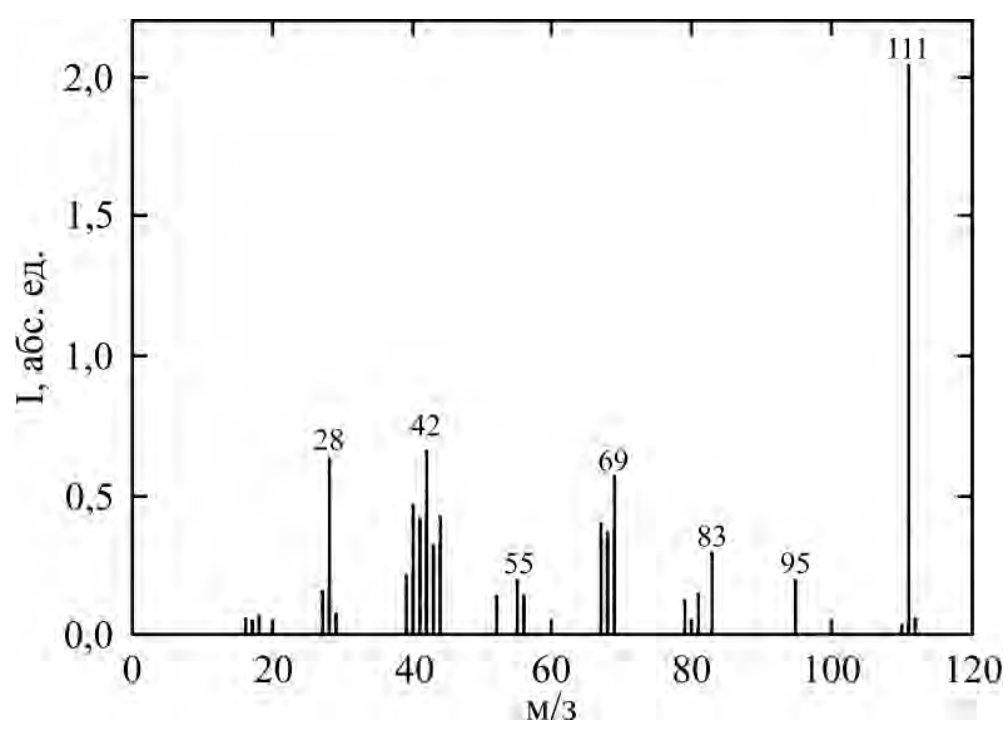

Рис. 1. Масс-спектр цитозина при энергии электронов 95 эВ.

где по оси абсцисс показано отношение массы иона к его заряду в системе атомных единиц, а по оси ординат - ток образованных ионов в произвольных единицах. Наиболее интенсивная линия в масс-спектре цитозина принадлежит молекулярному иону. Для фрагментации молекулярного иона необходим одновременный разрыв по крайней мере двух связей. Одновременный разрыв трех связей представляется менее вероятным. Мы полагаем, что наиболее вероятные разрывы должны быть в тех местах, для которых суммы двух связей являются самыми большими. С помощью метода AM1 нами были проведены теоретические расчеты длин связей в молекуле цитозина и ее молекулярном ионе. Для молекулярного иона цитозина, как это видно из рис. 2, в первую очередь следует выделить такие пары связей: 1 . N1 - C2, $\mathrm{C} 5-\mathrm{C} 6 ; 2$. N1 - C2, C2 - N3; 3. N1 - C2, C4 - C5; 4. N1-C2, N3-C4; 5. N3-C4, C4-C5.

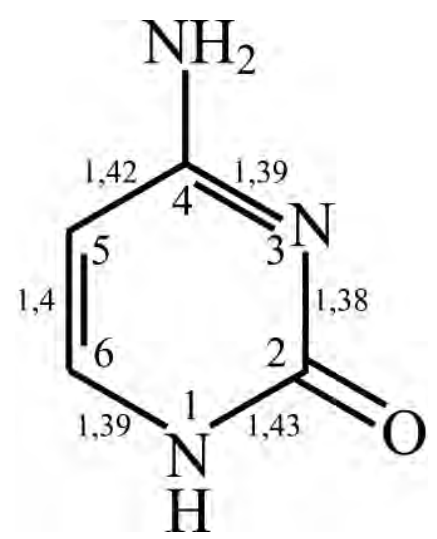

Рис. 2. Оптимизированная структура молекулярного иона цитозина.

Вероятности разрывов этих пар связей следует считать независимыми, а вновь образованные заряженные фрагменты могут быть источником дальнейшего распада. Таким образом, существуют независимые параллельнопоследовательные схемы фрагментации. Проиллюстрируем сказанное подробнее.

Разрывы связей N1 - C2, C5 - C6. При этом молекулярный ион $\mathrm{C}_{4} \mathrm{H}_{5} \mathrm{~N}_{3} \mathrm{O}^{+}$утрачивает нейтральный фрагмент $\mathrm{C}_{3} \mathrm{H}_{3} \mathrm{~N}_{2} \mathrm{O}(\mathrm{m} / z=85)$ и продуцируется заряженный фрагмент $\mathrm{CH}_{2} \mathrm{~N}^{+}$ $(m / z=28)$.

Разрывы связей N1 - C2, C2 - N3. Молекулярный ион $\mathrm{C}_{4} \mathrm{H}_{5} \mathrm{~N}_{3} \mathrm{O}^{+}$утрачивает нейтральный фрагмент $\mathrm{C}_{3} \mathrm{H}_{5} \mathrm{~N}_{3}(m / z=85)$ и также продуцирует заряженный фрагмент $\mathrm{CO}^{+}(m / z=28)$. В работе [17] возможность образования этого заряженного фрагмента не рассматривалась. Однако в спектре люминесценции цитозина под действием электронов была обнаружена интенсивная молекулярная полоса $\mathrm{CO}^{+}[18]$, что указывает на заметную вероятность разрывов связей $\mathbf{N 1}$ - C2, $\mathbf{C 2}$ - N3. Экспериментально разделить фрагменты $\mathrm{CH}_{2} \mathrm{~N}^{+}(m / z=28)$ и $\mathrm{CO}^{+}(m / z=28)$ невозможно, так как они имеют одинаковое отношение $\mathrm{m} / \mathrm{z}$. Однако теоретическое рассмотрение вышеприведенных схем фрагментации показывает, что образование фрагмента $\mathrm{CH}_{2} \mathrm{~N}^{+}$ $(\mathrm{m} / \mathrm{z}=28)$ будет преобладающим.

Разрывы связей N1 - C2, C4 - C5. Молекулярный ион $\mathrm{C}_{4} \mathrm{H}_{5} \mathrm{~N}_{3} \mathrm{O}^{+}$утрачивает нейтральный фрагмент $\mathrm{C}_{2} \mathrm{H}_{2} \mathrm{~N}_{2} \mathrm{O}(\mathrm{m} / z=70)$ и производит заряженный фрагмент $\mathrm{C}_{2} \mathrm{H}_{3} \mathrm{~N}^{+}(m / z=41)$, который, в свою очередь, последовательно теряя атомы водорода, продуцирует ионы $\mathrm{C}_{2} \mathrm{H}_{2} \mathrm{~N}^{+}(m / z=40)$ и $\mathrm{C}_{2} \mathrm{HN}^{+}(m / z=39)$.

Разрывы связей N1 - C2, N3 - C4. Разрыв этих связей запускает два конкурирующих процесса: а) молекулярный ион $\mathrm{C}_{4} \mathrm{H}_{5} \mathrm{~N}_{3} \mathrm{O}^{+}$ утрачивает нейтральный фрагмент $\mathrm{C}_{3} \mathrm{H}_{5} \mathrm{~N}_{2}$ $(m / z=69)$ и продуцирует заряженный фрагмент $\mathrm{CNO}^{+}(m / z=42)$; б) молекулярный ион $\mathrm{C}_{4} \mathrm{H}_{5} \mathrm{~N}_{3} \mathrm{O}^{+}$ утрачивает нейтральный фрагмент $\mathrm{CNO}$ 
Таблица. Абсолютные велнчнны сеченнй образовання положнтельных нонов молекул цнтознна и нх фрагментов прн энергнн электронов 95 эВ

\begin{tabular}{|c|c|c|}
\hline $\begin{array}{c}m / z, \\
\text { a.e M. }\end{array}$ & Ионы & $\sigma, 10^{-16} \mathrm{~cm}^{2}$ \\
\hline 111 & $\mathrm{C}_{4} \mathrm{H}_{5} \mathrm{~N}_{3} \mathrm{O}^{+}$ & 2,02 \\
\hline 95 & $\begin{array}{c}\mathrm{C}_{4} \mathrm{H}_{3} \mathrm{~N}_{2} \mathrm{O}^{+} ; \\
\mathrm{C}_{4} \mathrm{H}_{5} \mathrm{~N}_{3}^{+}\end{array}$ & 0,18 \\
\hline 83 & $\begin{array}{c}\mathrm{C}_{3} \mathrm{H}_{3} \mathrm{~N}_{2} \mathrm{O}^{+} ; \\
\mathrm{C}_{3} \mathrm{H}_{5} \mathrm{~N}_{3}^{+}\end{array}$ & 0,28 \\
\hline 69 & $\mathrm{C}_{3} \mathrm{H}_{5} \mathrm{~N}_{2}^{+}$ & 0,55 \\
\hline 68 & $\mathrm{C}_{3} \mathrm{H}_{4} \mathrm{~N}_{2}^{+}$ & 0,35 \\
\hline 67 & $\mathrm{C}_{3} \mathrm{HNO}^{+} ;$ & 0,38 \\
\hline 56 & $\mathrm{C}_{3} \mathrm{H}_{3} \mathrm{~N}_{2}^{+}$ & \\
\hline 55 & $\mathrm{C}_{2} \mathrm{H}_{2} \mathrm{NO}^{+} ;$ & 0,12 \\
$\mathrm{CN}_{2} \mathrm{O}^{+}$ & $\mathrm{C}_{2} \mathrm{H}_{3} \mathrm{~N}_{2}^{+}$ & 0,18 \\
\hline 52 & $\mathrm{C}_{3} \mathrm{H}_{2} \mathrm{~N}^{+}$ & 0,12 \\
\hline 44 & $\mathrm{CH}_{2} \mathrm{NO}^{+}$ & 0,4 \\
\hline 43 & $\mathrm{CHNO}^{+}$ & 0,3 \\
\hline 42 & $\mathrm{CH}_{2} \mathrm{~N}_{2}^{+} ;$ & 0,64 \\
\hline 41 & $\mathrm{CNO}^{+}$ & \\
\hline & $\mathrm{CHN}_{2}^{+} ;$ & 0,4 \\
\hline 40 & $\mathrm{C}_{2} \mathrm{H}_{3} \mathrm{~N}^{+}$ & \\
\hline 39 & $\mathrm{C}_{2} \mathrm{H}_{2} \mathrm{~N}^{+}$ & 0,45 \\
\hline 28 & $\mathrm{C}_{2} \mathrm{HN}^{+}$ & 0,2 \\
\hline 27 & $\mathrm{CO}^{+} ; \mathrm{CH}_{2} \mathrm{~N}^{+}$ & 0,62 \\
\hline & $\mathrm{CHN}^{+}$ & 0,14 \\
\hline
\end{tabular}

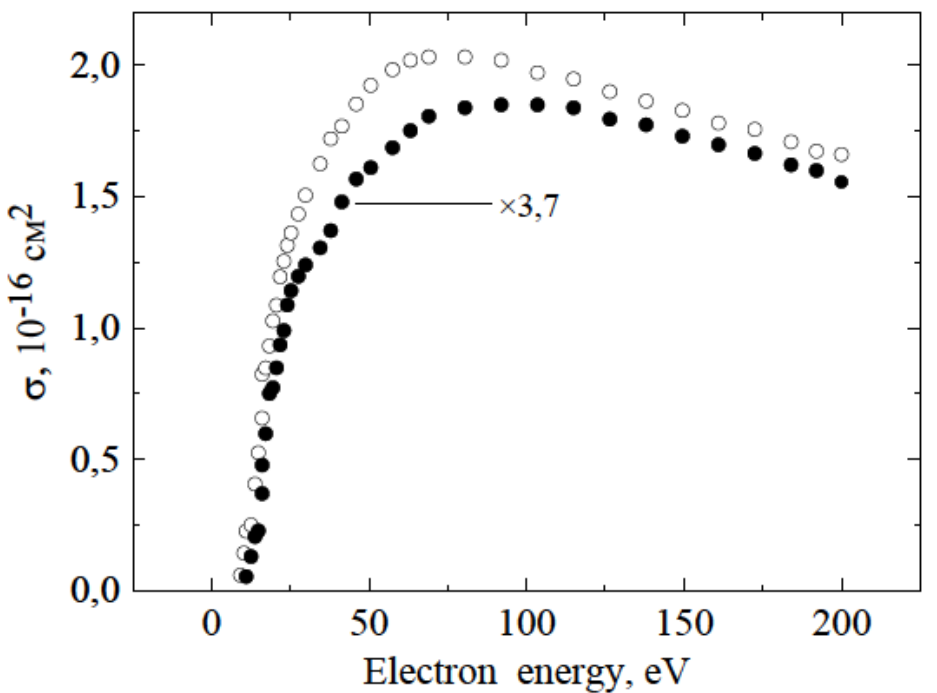

Рис. 3. Зависимости абсолютных величин сечений образования положительных ионов цитозина от энергии электронов: o- молекулярный ион $\mathrm{C}_{4} \mathrm{H}_{5} \mathrm{~N}_{3} \mathrm{O}^{+}(m / z=111) ; \bullet-$ ионный фрагмент $\mathrm{C}_{3} \mathrm{H}_{5} \mathrm{~N}_{2}^{+}(m / z=69)$.

$(m / z=42)$ и продуцирует заряженный фрагмент $\mathrm{C}_{3} \mathrm{H}_{5} \mathrm{~N}_{2}^{+} \quad(m / z=69)$. Вероятности обоих процессов, как это следует из масс-спектра цитозина, сопоставимы. В свою очередь заряженный фрагмент $\mathrm{C}_{3} \mathrm{H}_{5} \mathrm{~N}_{2}^{+}(m / z=69)$, последовательно теряя атомы водорода, продуцирует ионы $\mathrm{C}_{3} \mathrm{H}_{4} \mathrm{~N}_{2}^{+}(m / z=68)$ и $\mathrm{C}_{3} \mathrm{H}_{3} \mathrm{~N}_{2}^{+}(m / z=67)$.

Разрывы связей N1 - N3 - C4, C4 - C5. Молекулярный ион $\mathrm{C}_{4} \mathrm{H}_{5} \mathrm{~N}_{3} \mathrm{O}^{+}$утрачивает нейтральный фрагмент $\mathrm{CH}_{2} \mathrm{~N}(m / z=28)$ и производит заряженный фрагмент $\mathrm{C}_{3} \mathrm{H}_{3} \mathrm{~N}_{2} \mathrm{O}^{+}(\mathrm{m} / z=83)$.

Группа линий небольшой интенсивности с $m / z=52, m / z=55, m / z=56$, по-видимому, как это указано в работе [17], возникает в обратной Дильса-Альдера реакции $[19,20]$. Потеря молекулярным ионом $\mathrm{C}_{4} \mathrm{H}_{5} \mathrm{~N}_{3} \mathrm{O}^{+}$аминогруппы $\mathrm{NH}_{2} \quad(m / z=16)$ приводит $\kappa$ образованию заряженного фрагмента $\mathrm{C}_{4} \mathrm{H}_{3} \mathrm{~N}_{2} \mathrm{O}^{+}(m / z=95)$. 
Присутствие малоинтенсивных линий на приведенном масс-спектре в окрестности интенсивных линий можно трактовать как проявление изотопного состава ионов, а также отщепление атомов водорода от ионов в процессе их фрагментации.

Приведенные схемы представляются наиболее вероятными, однако не исключается ход фрагментации также по другим схемам.

Данные об абсолютных величинах парциальных сечений ионизации молекул цитозина приведены в таблице. Ее анализ показывает, что доминантным процессом в образовании положительных ионов цитозина является образование молекулярного иона (сечение по порядку величины $10^{-16} \mathrm{~cm}^{2}$ ), что свидетельствует о достаточной устойчивости цитозина к электронному удару. Это очень значимый факт для такой сложной молекулы, как цитозин, когда наибольшее парциальное сечение ионизации характеризует образование молекулярного иона. Множество сложных молекул не имеет устойчивых молекулярных ионов. Вместе с тем следует обратить внимание на величину суммарного сечения образования других фрагментов, превышающую в 2,9 раза сечение образования молекулярного иона.

Как видно из рис. 3, энергетическая зависимость сечения образования молекулярного иона в общих чертах (порог ионизации, энергии максимумов) похожа на энергетическую зависимость полного сечения ионизации [14]. Энергетический порог образования молекулярных

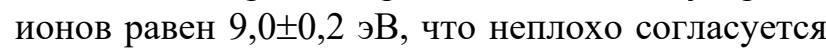
с данными, полученными другими методами $[21,22]$. А для заряженного фрагмента молекулы $\mathrm{C}_{3} \mathrm{H}_{5} \mathrm{~N}_{2}^{+}(m / z=69)$ порог его образования и положение максимального сечения сдвигаются в область более высоких энергий. В частности, порог образования ионного фрагмента цитозина $\left(\mathrm{C}_{3} \mathrm{H}_{5} \mathrm{~N}_{2}^{+}, \quad m / z=69\right)$ составляет $10,8 \pm 0,2$ эВ.

\section{ЗАКЛЮЧЕНИЕ}

Представлены возможные схемы фрагментации молекулы цитозина. Результаты квантовохимического расчета хорошо согласуются с данными эксперимента. Показано, что модельное описание фрагментации биомолекулы дает возможность разделить образование двух положительно заряженных фрагментов, имеющих одинаковое отношение $\mathrm{m} / z$.

Экспериментально определены абсолютные величины сечений образования положительных ионов биологически важной молекулы цитозина под воздействием медленных электронов.
Показано, что величина суммарного сечения образования положительно заряженных фрагментов в 3,7 раза превышает сечение образования молекулярного иона. Определение абсолютных значений сечений образования положительных ионов позволяет провести качественные оценки процессов, которые имеют место в биомолекулах. В частности, сечение ионизации биомолекулы в несколько раз больше сечения ионизации молекул воды $\mathrm{H}_{2} \mathrm{O}\left(1,5 \times 10^{-16} \mathrm{~cm}^{2}\right.$, согласно [23]), на долю которых приходится 70\% массы живых клеток. Отсюда следует, что протектирующие свойства молекул воды в условиях клетки будут выражены недостаточно. Ионизация нуклеотидных оснований, особенно диссоциативная ионизация, под влиянием вторичных низкоэнергетических электронов в свою очередь приводит к структурным и функциональным сдвигам в живых клетках. Прежде всего, следует ожидать нарушений стабильности системы водородных связей между комплементарными парами оснований, генотоксических и мутагенных изменений. Не исключено влияние на энергетику клетки, на протонные транспортные системы.

\section{ЛИТЕРАТУРА}

1. Суховия, М.И., Шафраньош, И.И., О возбуждении азотистых оснований нуклеиновых кислот низкоэнергетическими электронами, Tезисы докладов симпозиума «Механизмы радиационного поражения $u$ восстановления нуклеиновых кислот», Пущино, Научный центр биологических исследований АН СССР, 1980. С. 51.

2. Суховия, М.И., Славик, В.Н., Шафраньош, И.И., Шимон, Л.Л., Особенности взаимодействия молекул оснований нуклеиновых кислот с электронами малых энергий. Биополимеры $u$ клетка, 1991, т. 7, № 6, с. 77 .

3. Feil, S., Gluch, K., Matt-Leuber, S., Scheier, P. et al. Partial cross sections positive and negative ion formation following electron impact on uracil. J. Phys. $B, 2004$, vol. 37 , no. 15 , p. 3013 .

4. Aflatooni, K., Scheer, A.M., Burrow, P.D., Total dissociative electron attachment cross sections for molecular constituents of DNA, J. Chem. Phys., 2006, vol. 125, no. 5, p. 054301-1.

5. Афросимов, В.В., Басалаев, А.А., Морозов, Ю.Г., Панов, М.Н. и др. Фрагментация молекул аденина и урацила при захвате электронов в столкновении с ионами. ЖТФ, 2012, т. 82, № 5, с. 16.

6. Афросимов, В.В., Басалаев, А.А., Кузьмичев, В.В., Панов, М.Н. и др. Элементарные процессы при столкновениях ионов с молекулами триптофана, ЖТФ, 2016, т. 86, № 3, с. 25.

7. Ulysse, Pedreira-Segade, Cercile, Feuillie, Manuel, Pelletier, Laurent J., Michot et al. Adsorption of nucleotides onto ferromagnesian phyllosilicates: 
Significance for the origin of life, Geochim. Cosmochim. Acta, 2016, vol. 176, p. 81.

8. Шафраньош, И.И., Митропольский, И.Е., Кузьма, В.В., Свида, Ю.Ю. и др. Люминесценция урацила на поверхности керамики при электронном возбуждении. Журнал прикладной спектроскопии, 2018, т. 85, № 1, с. 32.

9. Sándor Góbi, Alexandre Bergantini, Ralf I., Kaiser. Degradation of Adenine on the Martian Surface in the Presence of Perchlorates and Ionizing Radiation: A Reflectron Time-of-flight Mass Spectrometric Study, The Astrophysical J., 2017, vol. 838, no. 2, p. 84.

10. Полякова, А.А., Молекулярный масс-спектральный анализ органических соединений. М.: Химия, 1993. 248 с.

11. Minaev, B.F., Shafranyosh, M.I., Svida, Yu.Yu., Sukhoviya, M.I. et al. Fragmentation of the adenine and guanine molecules induced by electron collisions, J. Chem. Phys., 2014, vol. 140, no. 17, p. 184303.

12. Shafranyosh, I.I., Sukhoviya, M.I., Shafranyosh, M.I., Shimon, L.L., Formation of positive and negative ions of thymine molecules under the action of slow electrons. Tech. Phys., 2008, vol. 53, no. 12, p. 1536.

13. Shafranyosh, I.I., Sukhoviya, M.I., Inelastic collisions of the uracil molecules with electrons. J. Chem. Phys., 2012, vol. 137, no. 18, p. 184303.

14. Shafranyosh, I.I., Sukhoviya, M.I., Shafranyosh, M.I., Absolute cross sections of positive and negative ion production in electron collision with cytosine molecules. J. Phys. B, 2006, vol. 39, no. 20, p. 4155.

15. Dewar, M.J.S., Thiel, W., The MNDO method. Approximations and parameters. J. Am. Chem. Soc., 1977, vol. 99, no. 15, p. 4899.

16. Dewar, M.J.S., Zoebish, E.G., Headly, E.E., Stewart, J.J., AM1: A general purpose quantum mechanical molecular model, J. Am. Chem. Soc., 1985, vol. 107, no. 13, p. 3902.

17. Jerry, M., Rice Gerald, O., Dudek and Michael Barber. Mass Spectra of Nucleic Acid Derivatives.
Pyrimidines, J. Am. Chem. Soc., 1965, vol. 87, no. 20, p. 4569.

18. Шафраньош, І.І., Свида, Ю.Ю., Суховія, М.І., Шкиндя, В.Ю. и др. Спектр свічення електричного розряду в парах цитозину. Науковий вісник УжНУ. Серія Фізика, 2016, т. 39, с. 88.

19. Diels, O., Alder, K. Synthesen in der hydroaromatischen Reihe. Justus Liebigs Annalen der Chemie, 1928, vol. 460, no. 1, p. 98.

20. Смит, В.А., Дильман, А.Д., Реакция ДильсаАльдера. Часть I. В кн..: Основы современного органического синтеза. М.: Бином. Лаборатория знаний, 2009, с. 605-642.

21. Lin, J., Yu, C., Peng, S., Akiyama, I. et al. Ultraviolet photoelectron studies of the ground-state electronic structure and gas-phase tautomerism of purine and adenine. J. Am. Chem. Soc., 1980, vol. 102, p. 4627.

22. Verkin, B.I., Yanson, I.K., Sukhodub, L.F., Teplicky, A.B., Biomolecular Interactions. New Experimental Approaches and Methods. Kiev: Naukova Dumka, 1985, 317 p.

23. Rapp, D., Englander-Golden, P., Total cross sections for ionization and attachment in gases by electron impact. I. Positive ionizations. J. Chem. Phys., 1965, vol. 43 , p. 1464.

\section{Summary}

The investigation of fragmentation processes in cytosine molecules caused by an electron impact was carried out using crossed electron and molecular beams and the AM1 quantum-chemical method. The probable schemes of cytosine fragmentation, absolute values of ionization partial sections and their energetic dependences are given. It is shown that the value of the total section of formation of positively charged fragments is 2.9 times larger than the section of the molecular ion formation. The calculations of the parameters of the cytosine molecule and its ions were performed.

Keywords: secondary electrons, cytosine, electron impact, ionization cross sections, energy dependences, fragmentation schemes, molecular ion 